\title{
On solvable minimally transitive permutation groups
}

\author{
Francesca Dalla Volta · Johannes Siemons
}

Received: 24 September 2006 / Revised: 15 April 2007 / Accepted: 18 April 2007 /

Published online: 14 June 2007

(C) Springer Science+Business Media, LLC 2007

\begin{abstract}
We investigate properties of finite transitive permutation groups $(G, \Omega)$ in which all proper subgroups of $G$ act intransitively on $\Omega$. In particular, we are interested in reduction theorems for minimally transitive representations of solvable groups.
\end{abstract}

Keywords Solvable permutation groups - Minimal transitivity

AMS Classifications $20 \mathrm{~B} 05 \cdot 20 \mathrm{D} 10 \cdot 20 \mathrm{D} 15$

\section{Introduction}

A finite permutation group $(G, \Omega)$ is minimally transitive if $G$ is transitive on $\Omega$ while all proper subgroups of $G$ are intransitive on $\Omega$. Evidently, any transitive permutation group contains minimally transitive subgroups acting on the same set and so this concept occurs naturally in reduction arguments. Closely related is the notion of minimally irreducible linear groups, namely those linear groups which act irreducibly on a vector space $V$ while all proper subgroups leave some proper subspace of $V$ invariant.

Solvable minimally transitive groups were first considered by Suprunenko [8] and Kopylova [3] who studied the groups of degree $p q$ with $p$ and $q$ primes. More recently Lucchini [4] studied minimal generating sets in minimally transitive groups, in connection with the asymptotic properties of permutation groups considered in Pyber [7]. In Ngo [6]

Work partially supported by M.I.U.R. and London Mathematical Society.

F. Dalla Volta

Dipartimento di Matematica e Applicazioni, Università Milano-Bicocca, Via R. Cozzi 53, 20125, Milano Italy

e-mail: Francesca.DallaVolta@unimib.it

J. Siemons $(\bowtie)$

School of Mathematics, University of East Anglia, Norwich, NR4 7TJ, UK

e-mail: j.siemons@uea.ac.uk 
non-regular metabelian minimally transitive groups are investigated, and Miller-Praeger [5] mention such groups in the context of vertex transitive graphs which are not Cayley graphs. A list of minimally transitive groups up to degree 30 is available in Hulpke [2], see also Conway et al. [1].

In this paper we consider solvable groups. Here in particular it happens frequently that a group action is not faithful. Therefore we study more generally arbitrary minimally transitive representations which may or may not be faithful. This language requires technical detail which could detract from the main matter; wherever possible we therefore try to stay close to the language of permutation groups which may appear more natural.

Any transitive permutation group contains minimally transitive subgroups and therefore it would be unreasonable to expect full classifications in general. However, under suitable restrictions some general results can be expected. For instance, for nilpotent groups there is a simple description of all their minimally transitive representations.

For a faithful action the primes dividing the order of a solvable group must divide the degree, see Theorem 3.1. In Sects. 2 and 3 we prove some reduction theorems for subgroups and factor groups. In particular, a construction is given to reduce a general minimally transitive action to the case where the degree contains only two primes. A good result is also available for actions of square-free degree, extending the work of Suprunenko and Kopylova.

\section{Minimally transitive groups}

Let $G \subseteq \operatorname{Sym} \Omega$ be a transitive permutation group on a finite set $\Omega$. Then $G$ is minimally transitive on $\Omega$ if every proper subgroup of $G$ is intransitive on $\Omega$. In the following we consider more generally an abstract finite group $G$ together with all its transitive actions, faithful or not. Thus if $A \subset G$ is a subgroup of $G$ let $G$ act on the cosets $G: A$ of $A$ in $G$. The kernel of this action is the core $K_{G: A}:=\bigcap_{g \in G} A^{g}$ of $A$ in $G$.

Thus $G$ acts minimally transitively on $G: A$ if and only if every subgroup $H$ with $K_{G: A} \subseteq H \subset G$ acts intransitively on $G: A$. It will be convenient to call such a subgroup $A$ an mt-stabilizer in $G$; we denote this as $A \subset_{\mathrm{m}} G$. Therefore $A \subset_{\mathrm{m}} G$ if and only if the following holds: Whenever $H \subseteq G$ is transitive on $G: A$ then $H K_{G: A}=G$. Evidently, if $A$ is an arbitrary subgroup of $G$ then $G / K_{G: A}$ always acts faithfully on $G: A$ and hence is a permutation group on $G: A$. This permutation group then is minimally transitive if and only if $A$ is an mt-stabilizer. For instance, if $A=1$ then $G$ is regular on $G: A$ and so $1 \subset_{m} G$. For another example suppose that $|G|=p q$ with distinct primes and Sylow subgroups $A \unlhd G$ and $B \unlhd G$. Then $K_{G: A}=A$ and $A \subset_{\mathrm{m}} G$ while $K_{G: B}=1$ and $B \not \not_{\mathrm{m}} G$.

\subsection{Preliminaries}

We begin by listing general properties of groups with minimally transitive action. For the remainder let $G$ be a finite group and let $A$ be a subgroup of $G$. The property of being an mt-stabilizer in $G$ is quite special as it relates to the subgroup as well as its embedding in $G$. Let $\mathcal{L}(G)$ denote the lattice of all subgroups of $G$. We will begin by describing some general properties of groups in $\mathcal{L}(G)$ which are mt-stabilizers in $G$. The next lemma is technical but essential; the first part we use later on without further mention.

Lemma 2.1 (i) Let $A \subseteq G$. Then $A \subset_{\mathrm{m}} G$ if and only if $A H=G$ for a subgroup $H \subseteq G$ implies that $H K_{G: A}=G$. 
(ii) Let $A \subset_{\mathrm{m}} G$ and let $B \subseteq A$. Then (a): $B \subset_{\mathrm{m}} G$ or $(b): K_{G: B} \neq K_{G: A}, B K_{G: A} \subset_{\mathrm{m}} G$ and there exists a subgroup $H \subseteq G$ with $H K_{G: B} \neq H K_{G: A}=G$. In particular, if $A \subset_{\mathrm{m}} G$ and $K_{G: A} \subseteq B \subseteq A$ then $B \subset_{\mathrm{m}} G$.

Proof

(i) Suppose that $A \subseteq G$ and that also $H \subseteq G$. Then $H$ is transitive on $G: A$ if and only if $A H=G$. Therefore by definition, if $A \varsigma_{\mathrm{m}} G$ and if $A H=G$ then $H K_{G: A}=G$. Conversely, if $A H=G$ implies that $H K_{G: A}=G$ then $H$ being transitive on $G: A$ means that $A H=G$ and so $H K_{G: A}=G$. Hence $A \subset_{\mathrm{m}} G$.

(ii) Assume that $A \subset_{\mathrm{m}} G$ and $B \subseteq A$, and suppose that $B \not \not_{\mathrm{m}} G$. Then there exists some $H$ such that $G=B H K_{G: B}$ but $G \neq H K_{G: B}$. As $K_{G: B} \unlhd K_{G: A}$ we have $G=A H K_{G: A}$. If $H K_{G: A} \neq G$ then $A \not \not_{\mathrm{m}} G$. Therefore $H K_{G: A}=G$ and so $K_{G: A} \neq K_{G: B}$. Next we compute the core $\bar{K}$ of $B K_{G: A}$ in $G$. Evidently, $K_{G: A} \subseteq \bar{K} \subseteq B K_{G: A} \subseteq A$ so that $\bar{K}=K_{G: A}$. If $B K_{G: A} \not_{\mathrm{m}} G$ then there exists a subgroup $\bar{H}$ such that $\left(B K_{G: A}\right) \bar{H}=G$ but $\bar{H} \bar{K} \neq G$. But then $A\left(K_{G: A} \bar{H}\right)=G$ and $\bar{H} K_{G: A} \neq G$, a contradiction. If $K_{G: A} \subseteq B \subseteq A$ then $K_{G: B}=K_{G: A}$ and hence the second alternative can not happen.

When dealing with the set of all mt-stabilizers in the subgroup lattice of $G$ the following is a useful notion: If $(\mathcal{L}, \leq)$ is any partially ordered set then a subset $\mathcal{M}$ of $\mathcal{L}$ is an order ideal in $\mathcal{L}$ if $X \in \mathcal{M}$ and $Y \leq X$ with $Y \in \mathcal{L}$ implies that $Y \in \mathcal{M}$.

Remark 2.2 From the last part of the lemma we deduce that the core-free mt-stabilizers in $G$ form an order ideal in the subgroup lattice $\mathcal{L}(G)$.

It is therefore often sufficient to know the 'top' mt-stabilizers, that is those which are maximal subject to being an mt-stabilizer. For instance, if $G$ is simple then the mt-stabilizers form an order ideal and this is described completely by its top elements. We may also ask when such top elements are maximal subgroups of $G$. Evidently, $A$ is maximal in $G$ precisely when $G$ acts primitively on $G: A$. More generally, $G$ acts quasi-primitively on $G: A$ if and only if any subgroup $N \neq K_{G: A}$ with $K_{G: A} \subseteq N \unlhd G$ acts transitively on $G$ : $A$. In particular, a transitive permutation group is quasi-primitive if all its normal subgroups $\neq 1$ are transitive.

Proposition 2.3 Let $G$ be quasi-primitive on $G:$. If $A \subset_{\mathrm{m}} G$ then $G / K_{G: A}$ is simple. Equivalently, if $(G, \Omega)$ is a quasi-primitive minimally transitive permutation group then $G$ is simple.

Proof Suppose that $A \subset_{\mathrm{m}} G$. If $G \unrhd N \supset K_{G: A}$ then $N$ is transitive on $G: A$ as $G$ is quasi-primitive on $G: A$. Hence $N=G$ as $A \subset_{\mathrm{m}} G$.

\subsection{A reduction theorem}

When studying minimal transitivity it is obviously useful to reduce a minimally transitive action $A G_{\mathrm{m}} G$ to one of a smaller group or to one of smaller degree. Minimal transitivity lends itself to good reduction arguments of this kind for normal subgroups. For this let $G$ be an arbitary finite group with an mt-stabilizer $A \varsigma_{\mathrm{m}} G$ of index $n$ in $G$. Let $H$ be a normal subgroup of $G$ with $K_{G: A} \subset H$ and $K_{G: A} \neq H \neq G$. Then $H$ is not transitive on $\Omega:=G: A$ and the orbits of $H$ on $\Omega$ are a system of imprimitivity for $G$. So these are of the shape $\Omega_{1}, \ldots, \Omega_{n^{*}}$ with $\left|\Omega_{i}\right|=s$ and $n^{*}:=\frac{n}{s}$. Let therefore $\Omega^{*}:=\left\{\Omega_{i} \mid i=1, \ldots, n^{*}\right\}$.

Let $\Omega_{1}$ be such that it contains the coset $1 A$ and let $B$ be the set-stabilizer of $\Omega_{1}$. In other words, $B=A H$ and in particular $H \subseteq K_{G: B}$. Now note that $B \subset_{\mathrm{m}} G$. For if $M \subseteq G$ satisfies 
$B M=G$ then $A H M=G$. As $A \subset_{\mathrm{m}} G$ we have $G=M H K_{G: A}$. But by choice, $K_{G: A} \subseteq H$ so that $G=M H$. As $H \subseteq K_{G: B}$ we get $G=M K_{G: B}$ and so $B \subset_{\mathrm{m}} G$. Equivalently, $G$ acts minimally transitively on $\Omega^{*}$. Therefore we have the following:

Theorem 2.4 Let $A \subset_{\mathrm{m}} G$ and suppose $H \neq G$ is normal in $G$ with $K_{G: A} \subset H \neq K_{G: A}$. Then $A \neq A H \subset_{\mathrm{m}} G$.

It is worth to formulate this statement in terms of permutation groups. In conjunction with Proposition 2.3 we have:

Theorem 2.5 Let $G$ be a minimally transitive permutation group on $\Omega$. If $G$ is quasi-primitive on $\Omega$ then $G$ is simple. Otherwise, if $H$ is a proper normal subgroup of $G$ then $G$ acts minimally transitively on the set of $H$-orbits.

In other words, a minimally transitive permutation group is either simple or otherwise induces a minimally transitive action on the orbits of any normal subgroup. Another kind of reduction occurs for the action of quotient groups, and this will be used later.

Lemma 2.6 Let $N$ be a normal subgroup of $G$ and let $N \subseteq A \subseteq G$. Then $A / N \subset_{\mathrm{m}} G / N$ if and only if $A \mathrm{Gm}_{\mathrm{m}} G$.

Proof If $N \subseteq A \subseteq G$ then $K_{G / N: A / N}=K_{G: A} / N$. Suppose that $A / N \subset_{\mathrm{m}} G / N$ but $A \not \not_{\mathrm{m}} G$. So there exist $H \subseteq G$ with $G=A H$ and $G \neq H K_{G: A}$. Consider $G / N=A / N \cdot H K_{G: A} / N$ and evaluate $H K_{G: A} / N \cdot K_{G / N: A / N}=H K_{G: A} / N \cdot K_{G: A} / N=H K_{G: A} / N \neq G / N$, a contradiction. Conversely, suppose that $A \subset_{\mathrm{m}} G$ but that $A / N \not \AA_{\mathrm{m}} G / N$. So there is a subgroup $N \subseteq H \subseteq G$ with $G / N=A / N \cdot H / N$ and $H / N \cdot K_{G: A} / N=H K_{G: A} / N \neq G / N$. So $G=A H$ with $H K_{G: A} \neq G$, a contradiction.

\section{Solvable groups}

For the remainder of the paper we shall restrict ourselves to minimally transitive representations of solvable groups. If $n$ is an integer let $\pi(n)$ be the set of primes dividing $n$. Similarly, $\pi(G)$ and $\pi(G: H)$ are the prime divisors in $|G|$ and $|G: H|$, respectively. Also, $|n|_{p}$ is the highest $p$-power dividing $n$.

The following theorem states the basic relation between $\pi(G)$ and the degree of any faithful minimally transitive action when $G$ is solvable. For nilpotent groups it completely characterizes all minimally transitive actions.

Theorem 3.1 (i) Let $A \subset_{\mathrm{m}} G$ such that $G / K_{G: A}$ is solvable. Then $\pi(G: A)=\pi\left(G / K_{G: A}\right)$. In particular, for a solvable minimally transitive permutation group $G$ of degree $n$ we have $\pi(G)=\pi(n)$.

(ii) Let $A \subset G$. If $A / K_{G: A}$ is contained in the Frattini subgroup of $G / K_{G: A}$ then $A \subset_{m} G$. Conversely, if $G / K_{G: A}$ is nilpotent and $A \in_{m} G$ then $A / K_{G: A}$ is contained in the Frattini subgroup of $G / K_{G: A}$.

(iii) If $A \subset_{\mathrm{m}} G$ and $|G: A|=p^{i}$ for some prime $p$ then $G / K_{G: A}$ is a proup and $A / K_{G: A}$ is contained in the Frattini subgroup of $G / K_{G: A}$.

Proof

(i) Let $H$ be a Hall $\pi(G: A)$-subgroup of $G$. Then $A H=G$ as the left hand side has order $|G|$. As $A \subset \mathrm{m} G$ therefore $H K_{G: A}=G$. As $K_{G: A} \subseteq A$ therefore $\pi(G: A)=\pi(G$ : $\left.K_{G: A}\right)$. 
(ii) Suppose that $A \not \not_{\mathrm{m}} G$. Then there exists some maximal subgroup $H^{\prime} \supseteq K_{G: A}$ with $G=A H^{\prime}$ and $H^{\prime} \neq G$. If in addition $A / K_{G: A}$ is contained in the Frattini subgroup of $G / K_{G: A}$ we have $A \subseteq H^{\prime}$, a contradiction. Conversely, if $G / K_{G: A}$ is nilpotent and if $H \supseteq K_{G: A}$ is a maximal subgroup of $G$ then $H$ is normal in $G$. Therefore $A H \supseteq K_{G: A}$ is a group and if $A \subset_{\mathrm{m}} G$ then $A H \neq G$, and hence $A \subseteq H$. (iii) This follows from (i) and (ii).

The next result is a general splitting principle reducing representations of non-nilpotent groups to representations of subgroups, generally involving fewer primes. We denote the Fitting subgroup of $X$ by $F(X)$.

Theorem 3.2 Let $G$ be a solvable group, suppose that $A \mathrm{Gm}_{\mathrm{m}} G$ is core-free and that $A$ is contained in $F=F(G)$. Let $\pi^{*}:=\pi(G: F)$ and let $Q$ be a Hall $\pi^{*}$-subgroup of $G$. Suppose that $P$ is a normal Sylow p-subgroup of $G$, let $A_{P}:=A \cap P$ and $A_{Q}:=A \cap Q$. Then $p$ does not belong to $\pi^{*}$. Furthermore, $A_{Q} \times A_{P}$ is core-free in $Q^{*} P$ and $A_{Q} \times A_{P} \subset_{\mathrm{m}} Q^{*} P$ for any conjugate $Q^{*}$ of $Q$.

Conversely, let $P_{1}, P_{2}, \ldots, P_{t}$ be the normal Sylow $p_{i}$-subgroups of $G$. Suppose there exist a subgroup $A_{Q}$ of $F \cap Q$ and subgroups $A_{P_{i}} \subseteq P_{i}$ such that $A_{Q} \times A_{P_{i}} \subset_{\mathrm{m}} Q^{*} P_{i}$ is core-free in $Q^{*} P_{i}$, for all conjugates $Q^{*}$ of $Q$ and all $i=1, \ldots, t$. Then $A_{Q} \times A_{P_{1}} \times \cdots \times A_{P_{t}} G_{\mathrm{m}} G$ is core-free in $G$.

For instance, in the simplest case when $\pi^{*}=\{q\}$ we may take $p$ to be any prime in $\pi(n) \backslash\{q\}$ where $n=|G: A|$. Then $Q \cap F$ is the Sylow $q$-subgroup of $F$ so that $A_{Q}$ is the Sylow $q$-subgroup of $A$. Similarly, $A_{P}$ is the Sylow $p$-subgroup of $A$ and hence $A_{Q} \times A_{P} \subset_{m} Q P$ is minimally transitive of degree $|n|_{q}|n|_{p}$. Note, for at least one choice of $p$ the group $Q P$ is not nilpotent, and evidently, groups of this type are at the basis of any induction in this case.

Proof Evidently, as $P$ is a normal Sylow subgroup of $G$ we have $P \subseteq F$ and so $p$ does not belong to $\pi^{*}$. Put $K:=K_{Q P:\left(A_{Q} \times A_{P}\right)}$. Then $K$ is centralized by every Sylow $r$-subgroup of $G$ for $r \neq p$ not dividing the order of $Q$. Further, it is normalized by $Q P$ and hence $K \subseteq A$ is a normal subgroup of $G$. As $A$ is core-free, $K$ is trivial. Now, suppose $A_{Q} \times A_{P} \not \mathrm{m} Q P$. Then there exists a subgroup $Y \subseteq Q P$ such that $\left(A_{Q} \times A_{P}\right) Y=Q P$ but $Y \neq Q P$. Let $S$ be the direct product of all Sylow $r$-subgroups of $F$ for $r \neq p$ not dividing the order of $Q$. This group is characteristic in $F$ and so normal in $G$. Therefore, $Y S$ is a group and $A(Y S)=(A Y) S \supseteq(Q P) S=Q F=G$. However, $Y S \neq G$. This is a contradiction, since $A \subset_{\mathrm{m}} G$. Finally note that $Q \cap F$ is normal in $F$. Thus if $Q$ is replaced by $Q^{f}$ then $A_{Q^{f}} \times A_{P} \subset_{\mathrm{m}} Q^{f} P$. But as $A \subseteq F$ we have $A \cap Q=A \cap Q^{f}$.

Conversely, let $A=A_{Q} \times A_{P_{1}} \ldots \times A_{P_{t}}$. Since $A_{Q} \times A_{P_{i}}$ are core-free in $Q P_{i}$ also $A$ is core-free in $G$. To show that $A \subset_{\mathrm{m}} G$ suppose that this is not the case. Let therefore $Y$ be a subgroup such that $G=A Y$ but $Y \neq G$. Thus $Y=Y^{*}\left(Y_{1} \times \ldots \times Y_{t}\right)$ for $Y_{i}:=Y \cap P_{i}$ and $Y^{*}$ a Hall $\pi^{*}$-subgroup of $Y$. Therefore $Y^{*} \subseteq Q^{f}$ for some $f \in F$.

Further, $G=\left(P_{1} \times \ldots \times P_{t}\right) Q^{f}$ and since $Y=\left(Y_{1} \times \ldots \times Y_{t}\right) Y^{*}$ we have $G=A Y=$ $\left(A_{Q} \times A_{P_{1}} \ldots \times A_{P_{t}}\right)\left(Y_{1} \times \ldots \times Y_{t}\right) Y^{*}$. As $A_{Q}$ is a $\pi^{*}$-subgroup of $F$ it centralizes all terms other than $Y^{*}$. Similarly, $Y_{i}$ centralizes all terms $A_{P_{j}}$ with $i \neq j$. Therefore we can rewrite this as $\left(P_{1} \times \ldots \times P_{t}\right) Q^{f}=\left(A_{P_{1}} Y_{1}\right) \times \ldots \times\left(A_{P_{t}} Y_{t}\right)\left(A_{Q} Y^{*}\right)$. For order reasons we have $A_{Q} Y^{*}=$ $Q^{f}$ and $A_{P_{i}} Y_{i}=P_{i}$ for $i=1, \ldots, t$. As $Y \neq G$ we have $Y \cap Q^{f} P_{r}=Y^{*} Y_{r} \neq Q^{f} P_{r}$ for at least one $r$, say $r=1$. Now consider $\left(A_{P_{1}} \times A_{Q}\right)\left(Y^{*} Y_{1}\right)=\left(A_{P_{1}} Y_{1}\right)\left(A_{Q} Y^{*}\right)=P_{1} Q^{f}$ This is a contradiction, since $A_{P_{1}} \times A_{Q} \subset_{\mathrm{m}} P_{1} Q^{f}$. 
3.1 Representations of square-free degree:

Next we turn to representations of square-free degree. Here we get precise information on the Fitting subgroup.

Theorem 3.3 For a solvable group $G$ suppose that $A \subset_{\mathrm{m}} G$ is core-free and has square-free index $n$ in $G$. Let $F$ be the Fitting group of $G$. Then $|F|$ is coprime to $|G: F|$ and all Sylow subgroups of $F$ are elementary abelian. In particular, $G$ is nilpotent if and only if $G$ is cyclic of order $n$, with $A=1$.

Let $\pi^{*}=\pi(n) \backslash \pi(F)$ and let $n^{*}$ be the product of the primes in $\pi^{*}$. If $C$ is a Hall $\pi^{*}$-subgroup of $A$ and if $Q$ is a Hall $\pi^{*}$-subgroup of $G$ containing $C$ then $|Q: C|=n^{*}$ and the action of $Q$ on $Q: C$ is permutationally equivalent to the action of $G$ on $G: A F$.

Proof If $n=p_{1} p_{2} \ldots p_{t}$ with pairwise distinct primes $p_{i}$ then $\pi(G)=\left\{p_{1}, p_{2}, \ldots, p_{t}\right\}$ by Theorem 3.1. Let $N \neq 1$ be a $p$-subgroup of $G$, say $p=p_{1}$, which is normal in $G$. We claim that $N$ is a Sylow subgroup of $G$. To prove this note that $N$ has $m:=\frac{n}{p}$ orbits $\Omega_{1}, \ldots, \Omega_{s}, \ldots, \Omega_{m}$ on $\Omega:=G: A$, all of length $\left|\Omega_{s}\right|=|N: N \cap A|=p$.

Let $S$ be a Sylow $p$-subgroup of $A N$. As $A N$ is the setwise stabilizer of the orbit $\Omega_{s}$ that contains $1 A$ we have that $p$ does not divide $|G: A N|$. Hence $S$ is a Sylow $p$-subgroup of $G$. If $Q$ is a Hall $p^{\prime}$-subgroup of $G$ then $S Q=G$ so that in particular $G=(A N) Q=A(N Q)$ for order reasons. As $A \subset_{\mathrm{m}} G$ we have $(N Q) K_{G: A}=G$ but as $A$ is core-free we have $N Q=G$. Therefore $N=S$ is a Sylow subgroup of $G$. For any $p \in \pi(F)$ let now $N$ be the unique Sylow $p$-subgroup of $F$. Thus $N$ is normal in $G$ and hence is a Sylow $p$-subgroup of $G$. It follows that $|F|$ is co-prime to $|G: F|$. By the same argument $N$ is characteristically simple and hence elementary abelian. Evidently, if $G=F$ is nilpotent then $G$ is abelian, hence regular on $\Omega$ and so cyclic of order $n=|\Omega|$.

As $|F|$ is co-prime to $|G: F|$ we may assume for the remainder that $Q$ is a $\pi^{*}$-subgroup of $G$ complementing $F$, with the further property that $Q \cap A=C$ is a $\pi^{*}$-subgroup of $A$. Then $G=Q F$ with $Q \cap F=1$ and $A F=C F$ with $C \cap F=1$ implies that the action of $G$ on the $n^{*}$ cosets of $A F$ in $G$ is permutationally equivalent to the action of $Q$ on the cosets of $C$. Hence $C \subset \mathrm{m} Q$ by Theorem 2.4.

Some comments are in order: (1) While the theorem could be formulated for permutation groups the resulting action of $G$ on the cosets of $A N$ is not faithful, and the same may be true for the action of $Q$ on the cosets of $C$.

(2) As $G$ is solvable there is at least one normal $p$-subgroup $N$, as in the proof, and for this $p$ it is the unique normal $p$-subgroup. This subgroup is elementary abelian, and $G$ acts irreducibly on it.

(3) The basis of induction for square free degrees occurs when $n$ is the product of two distinct primes. A complete analysis of the possibilities for $G$ can be found in Suprunenko [8] and Kopylova [3]. For the reader's benefit we collect their results here.

Theorem 3.4 (Suprunenko [8]) The permutation group $G$ is minimally transitive of degree $p q$, where $q<$ pare prime numbers with $q$ not dividing $(p-1)$, if and only if $G$ is isomorphic to one of the following

(i) the cyclic group of order pq,

(ii) a minimal non-abelian group $G=P Q$, where $|Q|=q$ and $P$ is normal in $G$, with $|P|=p^{m}$ where $m$ is the exponent of $p \bmod q$, or

(iii) a minimal non-abelian group $G=P Q$ with $Q$ is normal, $|P|=p$ and $|Q|=q^{r}$ where $r$ is the exponent of $q \bmod p$. 
The remaining case where $q^{r}$ with $r>0$ is the highest power of $q$ dividing $(p-1)$ is analyzed in Kopylova [3]. Here a similar description is obtained and it is shown that $G$ is (i) a group of order $q^{t} p$ with $0<t \leq r$; (ii) a group of order $q^{r+1} p^{q}$ or (iii) a group of order $p q^{l}$ where $l$ is the exponent of $q \bmod p$.

\subsection{Representations of degree involving two primes:}

From the discussion so far it is clear that $\{p, q\}$ groups and their minimally transitive representations play a special role. So let $A \subset \mathrm{m} G$ be core-free with $\pi(G)=\{p, q\}$. From Theorem 2.4 it is clear that any normal subgroup $N$ in $G$ gives rise to a minimally transitive representation $A N / N \subset_{\mathrm{m}} G / N$ of degree $\leq|G: A|$. Our first observation is the following

Lemma 3.5 Let $G$ be solvable and let $A \subset_{\mathrm{m}} G$ be core-free in $G$. Suppose that the prime $q$ divides $|G: A|$ to the first power only. Let $N$ be a q-group which is normal in $G$. Then $N$ is elementary abelian and is a Sylow subgroup, with $G$ acting irreducibly on $N$.

Proof Let $Q$ be a Sylow $q$-subgroup containing $N$ and let $P$ be a $q^{\prime}$-complement in $G=P Q$. Note that the $N$-orbits on $G: A$ are blocks of imprimitivity, all of equal size $q$ and $A N$ is the stabilizer of the orbit containing $1 A$. Therefore $q$ and $|G: A N|$ are co-prime so that $A N$ contains some Sylow $q$-subgroups of $G$. From this we have $(A N) P=G$, for order reasons. Since $A \subset_{\mathrm{m}} G$ and $A(N P)=G$ we have that $(N P) K_{G: A}=G$ but $K_{G: A}=1$ means $N P=G$. This says that $N$ is a Sylow $q$-subgroup of $G$ and hence $N=Q$. Next replace $N$ by a minimal normal subgroup of $G$. This group is elementary abelian. By the same argument $N$ has to be Sylow $q$-subgroup of $G$.

The lemma suggests that the natural choice for a normal subgroup is indeed the Fitting subgroup of $G$. We follow through with this process when $A$ has index $p^{i} q$. In this case, if either Sylow subgroup $S$ of $G$ is normal then $A S \subset_{\mathrm{m}} G$ gives a minimally transitive representation of a nilpotent group, and this situation is known from Theorem 3.1.

Otherwise none of the Sylow subgroups are normal and by Lemma $3.5 F_{1}=F(G)$ is a p-group. By Theorem 2.4 we have $A F_{1} G_{m} G$ and if $K \supseteq F_{1}$ is the core of $A F_{1}$ in $G$ then $A F_{1} / K G_{m} G / K$ is minimally transitive, faithful of degree $p^{j} q$ for $j<i$ and with $|G / K|_{p}<|G|_{p}$. If $F_{2}$ is the pre-image of $F(G / F)$ in $G$ then $F_{2} / F_{1}$ is a $q$-group. Thus, if $F_{2}$ is not contained in $K$ then Lemma 3.5 shows that $F_{2} K / K$ is a normal Sylow $q$-subgroup of $G / K$. In this case we are reduced to the nilpotent case. Otherwise $K \supseteq F_{2}$ so that $|G / K|_{q}<|G|_{q}$. The process stops when the group becomes nilpotent or when it is of Suprunenko-Kopylova type.

\section{References}

1. Conway JH, Hulpke A, McKay J (1998) On transitive permutation groups. LMS J Comput Math 1:1-8 (electronic)

2. Hulpke A The minimally transitive groups of degree up to 30, www.math.colostate.edu/ hulpke/paper/ctglist.pdf

3. Kopylova TI (1985) Solvable minimal transitive groups of permutations of degree $p q$. (Russian) VestsīAkad Navuk BSSR Ser Fīz-Mat Navuk 6:54-60, 126

4. Lucchini A (1998) Generating minimally transitive groups, in Groups and Geometries (Siena, 1996), 149-153, Trends Math, Birkhäuser, Basel, 1998

5. Miller AA, Praeger CE (1994) Non-cayley vertex-transitive graphs of order twice the product of two odd primes. J Algeb Combin 3: 77-111

6. Ngo DT (1976) Minimal transitive permutation groups. (Russian) VestīAkad Navuk BSSR Ser Fīz-Mat Navuk 6:5-14, 140 
7. Pyber L Asymptotic results for permutation groups. Groups and computation (New Brunswick, NJ, 1991), 197-219, DIMACS Ser Discrete Math Theoret Comput Sci 11, Amer Math Soc, Providence, RI, 1993

8. Suprunenko DA Solvable minimal transitive permutation groups of degree pq, Soviet Math Dokl (1986) 27:337-340; (Russian) Dokl Akad Nauk SSSR 269 (1983), 2:295-298Suprunenko DA Solvable minimal transitive permutation groups of degree pq, Soviet Math Dokl (1986) 27:337-340; (Russian) Dokl Akad Nauk SSSR 269 (1983), 2:295-298 